\title{
Diseño y evaluación del desempeño del algoritmo «iTIVA» para la administración manual de anestésicos intravenosos según objetivo en sitio efecto
}

\author{
David Eduardo Ramírez ${ }^{a, *}$ y José Andrés Calvache ${ }^{b, c}$ \\ a MD, Departamento de Anestesiología, Universidad del Valle, Cali, Colombia \\ ${ }^{\mathrm{b}}$ MD, MSc, FIPP, Departamento de Anestesiología, Universidad del Cauca, Popayán, Colombia \\ c Anesthesiology \& Biostatistics Departments, Erasmus University Medical Centre, Rotterdam, The Netherlands
}

\section{INFORMACIÓN DEL ARTÍCULO}

\section{Historia del artículo:}

Recibido el 23 de enero de 2015

Aceptado el 2 de febrero de 2016

On-line el 16 de marzo de 2016

\section{Palabras clave:}

Propofol

Farmacocinética

Anestesia

Simulación por computador

Bombas de infusión

\section{R E S U M E N}

Introducción: La administración de remifentanil y propofol con el uso de perfusores TCI ha mostrado beneficios en la práctica anestésica, sin embargo, su disponibilidad es limitada. Objetivo: Basados en modelos farmacocinéticos, diseñar un algoritmo capaz de calcular esquemas de infusión manual para alcanzar concentraciones en plasma y sitio efecto que puedan ser utilizados en bombas de infusión volumétricas. Adicionalmente, comparar la diferencia entre concentraciones deseadas y predichas mediante simulación farmacocinética.

Métodos: Siguiendo modelos de Minto y Schnider para remifentanil y propofol, respectivamente, el algoritmo se implementó en una aplicación llamada interactive TIVA (iTIVA) para los sistemas operativos iOS y Android. El desempeño del algoritmo se evaluó calculando esquemas de infusión para obtener distintas concentraciones en sitio efecto en la inducción y mantenimiento en 34 pacientes teóricos durante $240 \mathrm{~min}$.

Resultados: Los esquemas de infusión obtenidos para remifentanil y propofol presentaron una desviación sistemática promedio menor al $5 \%$ respecto a las concentraciones en sitio efecto objetivo en la inducción y mantenimiento. En la inducción una única infusión fue requerida para el remifentanil y propofol. Durante el mantenimiento, una única tasa de infusión fue requerida para el remifentanil y entre 2 y 5 cambios de tasa de infusión para el propofol para mantener una concentración estable. El algoritmo implementado en iTIVA calcula concentraciones similares que el software TivaTrainer ${ }^{\circledR}$.

Conclusiones: El desempeño del algoritmo para alcanzar concentraciones en sitio efecto durante la inducción y mantenimiento para remifentanil y propofol fue excelente con una desviación sistemática baja con respecto a las concentraciones objetivo deseadas.

(C) 2016 Sociedad Colombiana de Anestesiología y Reanimación. Publicado por Elsevier España, S.L.U. Este es un artículo Open Access bajo la licencia CC BY-NC-ND (http://creativecommons.org/licenses/by-nc-nd/4.0/).

* Autor para correspondencia. Departamento de Anestesiología, Universidad del Valle, Edificio 112, Hospital Universitario del Valle, piso 4. Cali, Colombia.

Correo electrónico: eramirez3@gmail.com (D.E. Ramírez). http://dx.doi.org/10.1016/j.rca.2016.02.002

0120-3347/@ 2016 Sociedad Colombiana de Anestesiología y Reanimación. Publicado por Elsevier España, S.L.U. Este es un artículo Open Access bajo la licencia CC BY-NC-ND (http://creativecommons.org/licenses/by-nc-nd/4.0/). 
Keywords:

Propofol

Pharmacokinetics

Anesthesia

Computer simulation

Infusion pumps
Design and performance evaluation of the "iTIVA" algorithm for manual infusion of intravenous anesthetics based on effect-site target

\section{A B S T R A C T}

Introduction: Remifentanil and propofol infusion using TCI pumps has proven to be beneficial for the practice of anesthesia but the availability of these systems is limited.

Objective: Designing a pharmacokinetic model-based algorithm for calculating manual infusion regimens to achieve plasma and effect-site concentrations that may be used in volume infusion pumps, and to compare the difference between the desired and the forecasted concentrations via pharmacokinetic simulation.

Methods: Using the Minto \& Schnider models for remifentanil and propofol respectively, the algorithm was implemented on an iTIVA application (interactive TIVA) for iOS and Android operating systems. The performance of the algorithm was evaluated estimating the infusion regimens for achieving different effect-site concentrations for induction and maintenance in 34 theoretical patients for 240 minutes.

Results: The infusion regimens obtained for remifentanil and propofol resulted in less than $5 \%$ average systemic deviation versus the target effect-site concentrations during induction and maintenance. Only one induction infusion was required for remifentanil and propofol. Just one infusion rate was required for remifentanil during maintenance, and between 2 to 5 infusion rate changes for propofol to maintain a stable concentration. The iTIVA-based algorithm estimates concentrations similar to the TivaTrainer ${ }^{\circledR}$ software.

Conclusions: The performance of the algorithm to achieve effect-site concentrations during induction and maintenance for remifentanil and propofol was excellent, with a low systemic deviation versus the desired target concentrations.

(c) 2016 Sociedad Colombiana de Anestesiología y Reanimación. Published by Elsevier España, S.L.U. This is an open access article under the CC BY-NC-ND license (http://creativecommons.org/licenses/by-nc-nd/4.0/).

\section{Introducción}

En la práctica actual de la anestesiología, los fármacos intravenosos son administrados utilizando dosificaciones estándar que se ajustan de manera empírica. Esta aproximación ignora la variabilidad interindividual de la cinética de los fármacos, la relación variable entre la dosis y la concentración en plasma y conlleva en algunas ocasiones a efectos adversos ${ }^{1}$.

En las últimas 2 décadas se han desarrollado modelos matemáticos - producto del estudio de las concentraciones plasmáticas de distintos fármacos- que describen el proceso de distribución y eliminación de los mismos. Estos han permitido predecir el efecto de administrar múltiples bolos, infusiones intravenosas o la combinación de ambos. El uso de estos modelos facilita la enseñanza y el aprendizaje de la farmacocinética aplicada a la anestesiología ${ }^{2,3}$.

La tecnología computacional ha facilitado la incorporación de los modelos teóricos para la simulación farmacocinética de fármacos como propofol (modelo de Schnider) ${ }^{4}$ y remifentanil (modelo de Minto) ${ }^{5}$. Estos sistemas permiten controlar las infusiones ajustándolas a determinadas concentraciones objetivo del fármaco deseado ${ }^{6-9}$. Esta tecnología se conoce en la actualidad como target control infusión (infusión controlada por objetivo, por sus siglas en inglés como TCI) y ha facilitado la administración de fármacos intravenosos como el remifentanil y propofol, permitiendo una rápida titulación para alcanzar distintos objetivos terapéuticos con mayor estabilidad hemodinámica ${ }^{3,10}$.
La disponibilidad de perfusores TCI en nuestra práctica diaria es limitada y pocos centros hospitalarios cuentan con estos, lo cual ha limitado la utilización de anestesia total intravenosa. Sin embargo, aun en países con libre acceso a la tecnología TCI, solo un 15-40\% administran TIVA con esta tecnología ${ }^{11-13}$ y restricciones de acceso en Estados Unidos de América o restricciones económicas, como en muchos países en desarrollo ${ }^{14,15}$, limitan su amplia difusión y uso. Por lo tanto, la administración manual de fármacos anestésicos con bombas de infusión volumétricas (BIV) es una práctica vigente.

En la actualidad, se han desarrollado sistemas de TCI pasivos que muestran la simulación farmacocinética en tiempo real de los fármacos administrados, los cuales han demostrado facilitar conseguir concentraciones estables en plasma y sitio efecto, con disminución en la carga mental para el anestesiólogo ${ }^{16-18}$. Estos sistemas solo se encuentran disponibles en ciertas máquinas de anestesia (p. ej., Navigator Applications Suite-GE Healthcare y SmartPilot View-Drager Medical), exigen una titulación cuidadosa pero no sugieren al profesional una tasa de infusión para alcanzar una concentración deseada ${ }^{19}$.

A diferencia de los sistemas de TCI pasivos, los perfusores TCI alcanzan concentraciones en plasma y sitio efecto de una manera rápida y precisa con el uso de complejos esquemas de infusión que varían cada $10 \mathrm{~s}$ controlados por un microprocesador según un modelo farmacocinético, manteniendo concentraciones estables en el tiempo ${ }^{20}$. Hasta el momento, no es posible emular el desempeño de los perfusores TCI con 
un método manual, dadas la alta complejidad matemática y la variación estrecha de las tasas de infusión.

Utilizando los modelos descrito por Minto et al. ${ }^{5}$ y Schnider et al. ${ }^{4}$ - para remifentanil y propofol, respectivamentees posible alcanzar y mantener concentraciones en plasma y sitio efecto utilizando un algoritmo matemático que permita una pequeña -y tolerable- variación en dichas concentraciones. Basados en esta hipótesis, el objetivo de este estudio fue diseñar un algoritmo capaz de calcular esquemas de infusión manual para alcanzar concentraciones en plasma y sitio efecto, y comparar la diferencia entre las concentraciones deseadas y las predichas mediante simulación farmacocinética.

\section{Metodología}

Este estudio fue desarrollado en 2 fases: primero se obtuvo la información teórica sobre el comportamiento farmacocinético de los fármacos anestésicos intravenosos, con lo cual se desarrolló un algoritmo para simular concentraciones en plasma y sitio efecto, y calcular esquemas de infusión para alcanzar una concentración en plasma y sitio efecto específicas. Este algoritmo se implementó en una aplicación móvil llamada «iTIVA» (del inglés, interactive TIVA, TIVA interactiva), para los sistemas operativos iOS y Android.

En la segunda fase se usó la simulación de los esquemas de infusión calculados a partir del algoritmo desarrollado como método para evaluación, como ha sido descrito en trabajos previos $^{21}$.

\section{Desarrollo del algoritmo}

La mayoría de los modelos de los anestésicos intravenosos como el remifentanil y propofol están basados en modelos tricompartimentales ${ }^{2,3}$, que son descritos por la ecuación 1, que predice la concentración en plasma luego de un bolo «d» en un tiempo $« \mathrm{t}{ }^{21}$ :

$C_{\text {bolus }}(d . t)=d\left(A \cdot e^{-\alpha t}+B \cdot e^{-\beta t}+C \cdot e^{-\gamma t}\right)$

La concentración plasmática de una infusión continua a una dosis «d» es igual a la repetición infinitesimal de bolos «di» equivalentes a «d» en un tiempo «t», lo cual es descrito por la integral de la ecuación 1:

$C_{\text {infusion }}(d, t)=d \int_{0}^{\infty} C_{\text {bolo }}(t) d t$

Al despegar «d» de la ecuación 2, se obtiene la dosis de infusión «d» para alcanzar una concentración plasmática en un tiempo «t».

$d\left(c_{\text {plasma }}, t\right)=\frac{{ }^{c_{\text {plasma }}}}{\int_{\infty}{ }^{0} C_{\text {bolo }}(t) d t}$
Cuando se varían las infusiones en el tiempo, la diferencia entre la nueva infusión «dn» en un tiempo «t $+\mathrm{n}$ » se adiciona a la primera infusión, como se describe en la ecuación 4:

concentració $n(d, t+n)=d C_{\text {objetivo }}(t+n)+\left(d_{n}-d\right) C_{\text {objetivo }}(t)$

Las concentraciones en sitio efecto se obtienen a partir de las concentraciones plasmáticas a través de una solución no analítica explicado en la sección de códigos en el sitio web http://www.opentci.org/

Con la ecuación 3 se obtiene una velocidad de infusión «d» para alcanzar concentraciones en plasma «Cp» en un tiempo «t», a partir de esa velocidad de infusión «d», se aumenta «d» iterativamente en 0,1 , hasta obtener la dosis «d» para alcanzar la concentración en sitio efecto objetivo.

Posteriormente, debemos calcular una infusión de mantenimiento que compense las pérdidas por distribución y eliminación, para mantener un estado seudoestable para una concentración deseada. Para lo cual la ecuación 5 permite calcular una tasa de infusión «d» en un tiempo «t», la cual compensa en ese momento las pérdidas por distribución y eliminación, la cual se mantiene por un tiempo «t $+\mathrm{n} »$, hasta que la concentración en plasma simulada supere la concentración objetivo «C» $+0,1$, en ese momento se recalcula una nueva infusión de mantenimiento en un tiempo «t $+\mathrm{n}$ », con la ecuación 5 , y así sucesivamente durante el tiempo que deseemos mantener la concentración.

$$
\begin{aligned}
& \text { Infusión de mantenimiento }\left(\mathrm{C}_{\text {objetivo }}, \mathrm{t}\right) \\
& \quad=\mathrm{C}_{\text {objetivo }} \cdot \mathrm{V}_{1} \cdot\left(k_{10}+k_{12} \cdot e^{-k_{21} t}+k_{13} \cdot e^{-k_{31} t}\right)
\end{aligned}
$$

Durante el mantenimiento no hay diferencia cuando el objetivo es plasma o sitio efecto, dado que ambos compartimientos tienden a equilibrarse rápidamente durante esta etapa.

\section{Desempeño del algoritmo}

Para evaluar el desempeño de iTIVA en obtener concentraciones en sitio efecto a partir de un esquema de infusión manual, se usaron datos de 34 pacientes teóricos. A partir de estos, se calcularon esquemas de infusión para un tiempo de $240 \mathrm{~min}$, para obtener diferentes concentraciones en sitio efecto, asignadas de manera aleatoria para remifentanil y propofol. Los datos utilizados se muestran en la tabla 1.

Los datos fueron tabulados en Microsoft Excel 2011 (Redmond, EE. UU.) y exportados al paquete estadístico R para su análisis y manejo gráfico ${ }^{22}$. Las medias \pm desviaciones estándar de las concentraciones simuladas fueron calculadas para cada minuto y los datos se agruparon de acuerdo con las concentraciones objetivo en sitio efecto.

Para evaluar la precisión de las concentraciones predichas por iTIVA se calcularon el error predictivo, la tasa de error predictivo y la desviación sistemática (o sesgo) con respecto a la concentración deseada (tabla 2). Esta metodología fue previamente descrita por Lerou y Booij ${ }^{23}$. Estos resultados se presentan de forma gráfica mediante estadísticos de resumen y de dispersión. El desempeño del algoritmo se 
Tabla 1 - Datos antropométricos de los pacientes simulados $(\mathbf{n}=34)$

\begin{tabular}{|c|c|c|c|c|c|c|c|c|}
\hline \multirow{3}{*}{ Sexo } & \multirow{3}{*}{ Edad (años) } & \multirow{3}{*}{ Peso (kg) } & \multirow{3}{*}{ Altura $(\mathrm{cm})$} & \multirow{3}{*}{ IMC $\left(\mathrm{kg} / \mathrm{m}^{2}\right)$} & \multirow{2}{*}{\multicolumn{2}{|c|}{$\begin{array}{l}\text { Remi }^{\mathrm{a}} \\
(\mathrm{ng} / \mathrm{ml})\end{array}$}} & \multirow{2}{*}{\multicolumn{2}{|c|}{$\begin{array}{l}\text { Propo }^{a} \\
(\mu \mathrm{g} / \mathrm{ml})\end{array}$}} \\
\hline & & & & & & & & \\
\hline & & & & & I & $\mathrm{M}$ & I & $\mathrm{M}$ \\
\hline F & 77 & 88 & 166 & 31,9 & 7,0 & 5,0 & 3,0 & 2,5 \\
\hline $\mathrm{F}$ & 70 & 84 & 174 & 27,7 & 5,0 & 4,0 & 3,0 & 2,0 \\
\hline F & 46 & 91 & 166 & 33,0 & 7,0 & 5,0 & 3,0 & 2,0 \\
\hline F & 72 & 76 & 155 & 31,6 & 5,0 & 4,0 & 3,0 & 2,5 \\
\hline F & 55 & 68 & 170 & 23,5 & 7,0 & 5,0 & 3,0 & 2,0 \\
\hline F & 51 & 58 & 161 & 22,4 & 7,0 & 5,0 & 3,5 & 3,0 \\
\hline $\mathrm{F}$ & 21 & 56 & 160 & 21,9 & 7,0 & 5,0 & 3,0 & 2,5 \\
\hline $\mathrm{F}$ & 33 & 62 & 156 & 25,5 & 7,0 & 5,0 & 3,5 & 3,0 \\
\hline $\mathrm{F}$ & 36 & 54 & 158 & 21,6 & 5,0 & 4,0 & 3,0 & 2,0 \\
\hline F & 28 & 68 & 156 & 27,9 & 5,0 & 4,0 & 3,0 & 2,5 \\
\hline F & 61 & 71 & 174 & 23,5 & 7,0 & 5,0 & 3,5 & 3,0 \\
\hline F & 31 & 88 & 180 & 27,2 & 7,0 & 5,0 & 3,5 & 3,0 \\
\hline F & 65 & 90 & 182 & 27,2 & 5,0 & 4,0 & 3,0 & 2,0 \\
\hline M & 30 & 81 & 186 & 23,4 & 7,0 & 5,0 & 3,0 & 2,5 \\
\hline M & 27 & 79 & 171 & 27,0 & 7,0 & 5,0 & 3,5 & 3,0 \\
\hline M & 41 & 102 & 188 & 28,9 & 7,0 & 5,0 & 3,5 & 3,0 \\
\hline M & 75 & 61 & 158 & 24,4 & 5,0 & 4,0 & 3,0 & 2,0 \\
\hline M & 26 & 110 & 173 & 36,8 & 7,0 & 5,0 & 3,0 & 2,5 \\
\hline M & 72 & 79 & 167 & 28,3 & 5,0 & 4,0 & 3,0 & 2,0 \\
\hline $\mathrm{M}$ & 31 & 64 & 177 & 20,4 & 7,0 & 5,0 & 3,5 & 3,0 \\
\hline $\mathrm{M}$ & 25 & 88 & 178 & 27,8 & 5,0 & 4,0 & 3,0 & 2,0 \\
\hline $\mathrm{M}$ & 62 & 70 & 170 & 24,2 & 5,0 & 4,0 & 3,0 & 2,5 \\
\hline $\mathrm{M}$ & 22 & 70 & 168 & 24,8 & 7,0 & 5,0 & 3,5 & 3,0 \\
\hline $\mathrm{M}$ & 44 & 72 & 162 & 27,4 & 7,0 & 5,0 & 3,5 & 3,0 \\
\hline $\mathrm{M}$ & 53 & 86 & 150 & 38,2 & 5,0 & 4,0 & 3,0 & 2,0 \\
\hline $\mathrm{M}$ & 66 & 72 & 164 & 26,8 & 5,0 & 4,0 & 3,0 & 2,5 \\
\hline $\mathrm{M}$ & 27 & 62 & 154 & 26,1 & 7,0 & 5,0 & 3,0 & 2,5 \\
\hline
\end{tabular}

F: femenino; M: masculino.

a Concentraciones objetivo para remifentanil (Remi) y propofol (Propo) durante la inducción (I) y mantenimiento (M). Fuente: autores.

Tabla 2 - Definición de las variables para estimación del desempeño

\begin{tabular}{llll} 
Variable & Unidad & $\begin{array}{c}\text { Definición } \\
\text { matemática }\end{array}$ & Definición \\
\hline Error predicho (ep) & No aplica & $\begin{array}{l}e p=C p-C d \\
\% e p=\frac{C p-C d}{C d} \times 100\end{array}$ & $\begin{array}{l}\text { Diferencia entre las concentraciones predichas y deseadas } \\
\text { Porcentaje de error entre las concentraciones predichas y deseadas }\end{array}$ \\
Porcentaje de error predicho (\%ep) & $\%$ & Desviación sistemática del error total, con dirección (signo) y magnitud \\
Desviación sistemática (DS) & $\%$ & $\mathrm{DS}=\frac{1}{n} \sum_{i=1}^{n} \% e p$ &
\end{tabular}

Cd: concentración deseada; Cp: concentración predicha.

Fuente: adaptada de Lerou et al..$^{23}$.

analizó de manera independiente para obtener concentraciones objetivo durante la inducción y mantenimiento. Por definición, se consideró la inducción como el periodo comprendido entre el quinto y sexto minutos, y el mantenimiento como el periodo entre el minuto 7 hasta la finalización de la simulación (minuto 240).

Finalmente, las concentraciones obtenidas en plasma y sitio efecto mediante iTIVA se compararon con las concentraciones calculadas con el software TivaTrainer ${ }^{\circledR}$ (versión 8; European Society for Intravenous Anaesthesia, Glasgow, Reino Unido) (tabla 3). Dados los resultados post hoc, no se realizaron contrastes de hipótesis en esta comparación.

\section{Resultados}

El algoritmo se implementó exitosamente en una aplicación para iOs y Android, la cual se encuentran disponible de manera gratuita en AppStore y Google Play. La figura 1 muestra diferentes pantallas de la aplicación para iPhone, entre ellas, ingreso de datos antropométricos, las 
Tabla 3 - Concentraciones en plasma y sitio efecto para propofol y remifentanil al simular distintos esquemas de infusión con iTIVA vs. TivaTrainer ${ }^{\circledR}$
iTIVA

\begin{tabular}{llllll} 
& \multicolumn{2}{c}{ iTIVA } & & \multicolumn{2}{c}{ TivaTrainer $^{\circledR}$} \\
\cline { 2 - 3 } \cline { 5 - 6 } Tiempo (min) & Plasma & Sitio efecto & & Plasma & Sitio efecto \\
\hline Propofol & & & & \\
Minuto 5 & $3,0 \pm 0,7$ & $2,4 \pm 0,5$ & & $3,0 \pm 0,7$ & $2,4 \pm 0,5$ \\
Minuto 10 & $2,2 \pm 0,6$ & $2,2 \pm 0,6$ & & $2,2 \pm 0,6$ & $2,2 \pm 0,6$ \\
Minuto 30 & $2,3 \pm 0,5$ & $2,4 \pm 0,5$ & & $2,3 \pm 0,5$ & $2,4 \pm 0,5$ \\
Minuto 60 & $2,4 \pm 0,5$ & $2,4 \pm 0,5$ & & $2,4 \pm 0,5$ & $2,4 \pm 0,5$ \\
Minuto 120 & $2,3 \pm 0,5$ & $2,4 \pm 0,5$ & & $2,3 \pm 0,5$ & $2,4 \pm 0,5$ \\
Minuto 180 & $2,4 \pm 0,5$ & $2,4 \pm 0,5$ & & $2,4 \pm 0,5$ & $2,4 \pm 0,5$ \\
Minuto 240 & $2,3 \pm 0,5$ & $2,4 \pm 0,5$ & & $2,3 \pm 0,5$ & $2,4 \pm 0,5$ \\
Remifentanil & & & & \\
Minuto 5 & $6,5 \pm 2,9$ & $5,2 \pm 2,3$ & & $6,5 \pm 2,9$ & $5,2 \pm 2,3$ \\
Minuto 10 & $4,0 \pm 1,7$ & $4,3 \pm 1,8$ & & $4,0 \pm 1,7$ & $4,3 \pm 1,8$ \\
Minuto 30 & $4,1 \pm 1,8$ & $4,1 \pm 1,7$ & & $4,1 \pm 1,8$ & $4,1 \pm 1,7$ \\
Minuto 60 & $4,1 \pm 1,8$ & $4,1 \pm 1,8$ & & $4,1 \pm 1,8$ & $4,1 \pm 1,8$ \\
Minuto 120 & $4,2 \pm 1,8$ & $4,2 \pm 1,8$ & & $4,2 \pm 1,8$ & $4,2 \pm 1,8$ \\
Minuto 180 & $4,2 \pm 1,8$ & $4,2 \pm 1,8$ & & $4,2 \pm 1,8$ & $4,2 \pm 1,8$ \\
Minuto 240 & $4,2 \pm 1,8$ & $4,2 \pm 1,8$ & & $4,2 \pm 1,8$ & $4,2 \pm 1,8$ \\
\hline
\end{tabular}

Los datos se presentan como media \pm desviación estándar (DE).
Fuente: autores. concentraciones objetivo, el esquema de infusión calculado y la visualización gráfica del comportamiento farmacocinético y farmacodinámico ${ }^{24}$ en el tiempo.

Para poder mantener una concentración estable durante la inducción, se requirió una única velocidad de infusión para remifentanil y propofol. Para mantener una concentración estable durante los $235 \mathrm{~min}$ del mantenimiento, se necesitó una única velocidad de infusión para remifentanil y entre 2 y 5 variaciones de la velocidad de infusión para propofol (moda $=4$ ).

Las concentraciones deseadas y predichas para plasma y sitio efecto resultado de la simulación y organizadas por objetivos deseados para propofol y remifentanil se presentan en sentan un énfasis en los minutos iniciales correspondientes a la inducción anestésica.

La figura 4 presenta la desviación sistemática versus el tiempo de las concentraciones predichas para propofol y remifentanil por el algoritmo en comparación con las deseadas por el operador.

Las concentraciones en plasma y sitio efecto, así como su variabilidad al simular distintos esquemas de infusiones con el algoritmo implementado en iTIVA versus las calculadas con el software TivaTrainer ${ }^{\circledR}$, fueron similares para diferentes tiempos de evaluación (tabla 3).

\section{Discusión}

Los principales resultados de este estudio son: 1) se desarrolló e implementó el algoritmo descrito de forma exitosa; 2) el desempeño del algoritmo propuesto para alcanzar concentraciones en sitio efecto durante la inducción y mantenimiento para remifentanil y propofol fue excelente; 3) el algoritmo presenta una variabilidad tolerable con respecto a las las figuras 2 y 3 respectivamente. Los ejes de tiempo (eje X) pre-

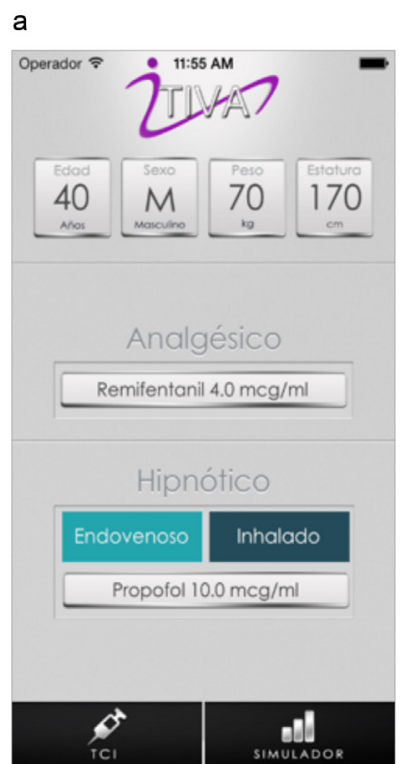

b

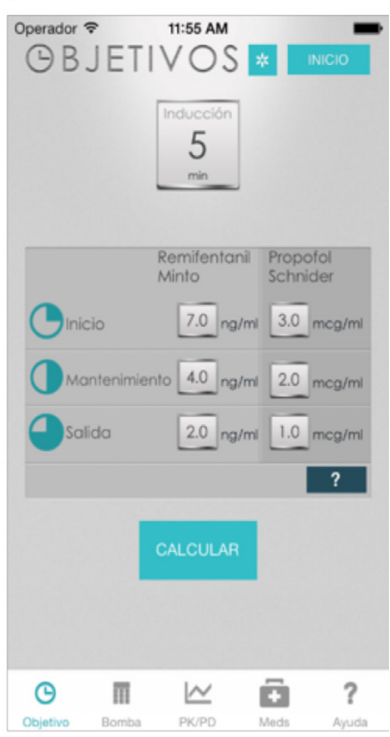

C

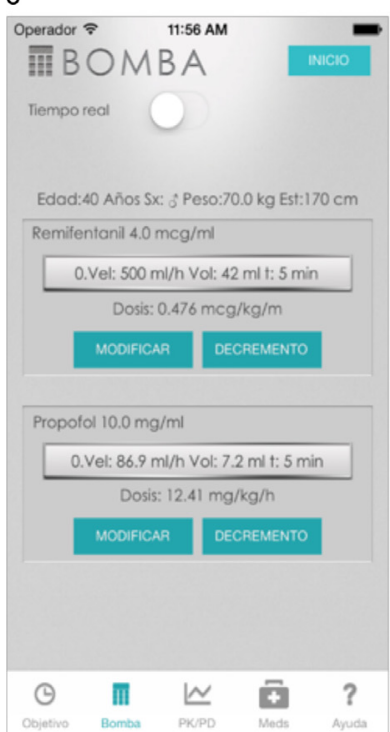

d

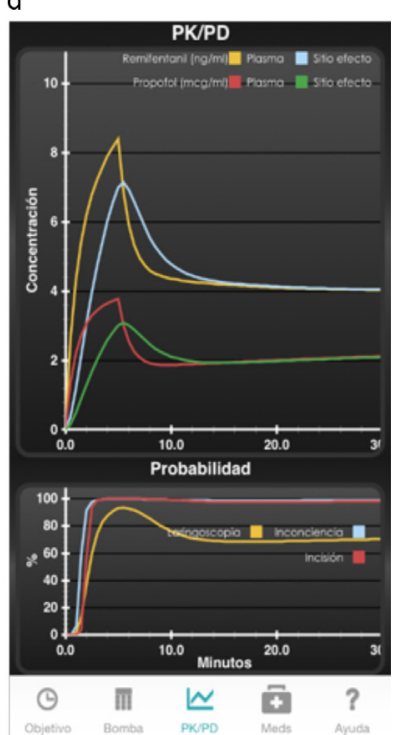

Figura 1 - Pantallas de la aplicación iTIVA para iPhone (a, b, c, d).

Fuente: autores.

concentraciones deseadas la cual se reduce en el periodo de mantenimiento, y 4) el algoritmo implementado en iTIVA para simular concentraciones en plasma y sitio efecto según un esquema de infusión se comporta de forma similar que el software TivaTrainer ${ }^{\circledR}$.

\section{Desarrollo e implementación del algoritmo}

La implementación del algoritmo desarrollado se hizo sin mayores dificultades con la ayuda de herramientas de desarrollo de las compañías Apple y Google para sus sistemas iOs (Xcode) y Android (Eclipse), respectivamente. Inicialmente, el algoritmo se implementó para Android dado que ofrece menores restricciones para la publicación en su tienda de aplicaciones (este hecho facilitó la depuración del código de 

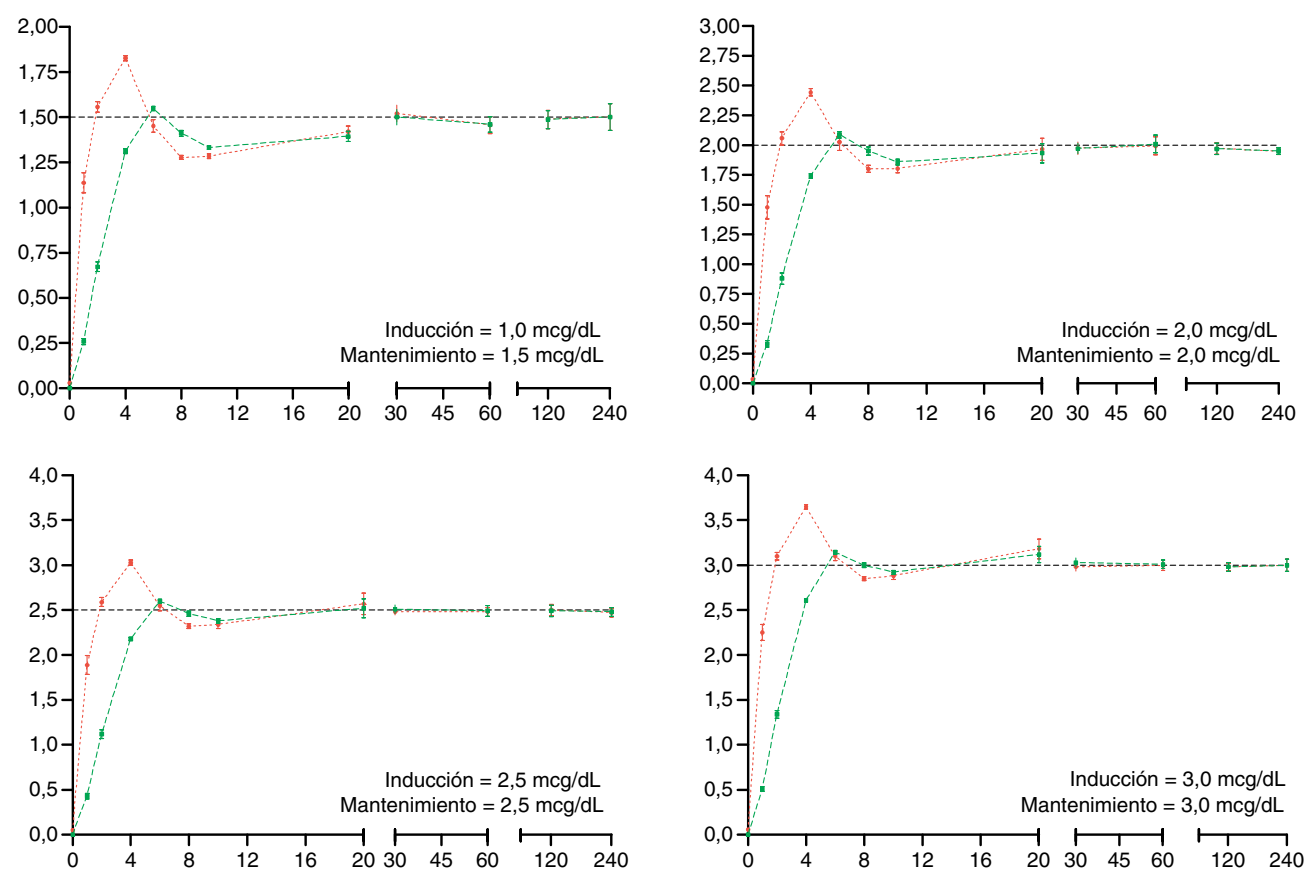

Plasma $\rightarrow$ Sitio efecto

Figura 2 - Concentraciones deseadas y predichas para plasma y sitio efecto organizadas por objetivos deseados para propofol.

Fuente: autores.

programación). Posteriormente, se adaptó al sistema operativo iOS aprovechando las similitudes en la sintaxis entre los lenguajes de programación (Java y $\mathrm{C}++$ ).

Para el óptimo desempeño de este algoritmo (durante 240 min de simulación), se requieren entre 200.000 y 250.000 cálculos matemáticos. En la actualidad, esto no representa mayores limitaciones para los microprocesadores disponibles aún en dispositivos móviles básicos.

\section{Desempeño del algoritmo y variabilidad}

Durante el periodo de inducción anestésica (minuto 5 y 6) la desviación sistemática de las concentraciones deseadas de los 2 medicamentos fue inferior al 5\%. Esta baja variabilidad se traduce como una proximidad alta entre la concentración deseada y la alcanzada por el algoritmo.

Durante el mantenimiento (minuto 7 a 240), los esquemas de infusión mostraron cambios en la desviación sistemática, que en general fue inferior al 5\% para propofol y el 15\% para remifentanil. Esta variabilidad se reduce a significativamente en el minuto 20 para remifentanil y en el 30 para propofol. La divergencia observada en los primeros minutos se puede relacionar al cambio de una concentración objetivo mayor a una menor. Durante este periodo -y para mantener una concentración estable con variación inferior al 5\%- se requirió una única tasa de infusión para remifentanil y entre 2 y 5 cambios progresivos en la tasa de infusión de propofol. Esta diferen- cia puede explicarse por las propiedades farmacocinéticas del primero que lo hacen insensible al contexto ${ }^{25}$.

A diferencia de los esquemas de infusión usados por perfusores TCI que requieren aproximadamente 1.400 cambios de la tasa de infusión durante 240 minutos, este algoritmo permite obtener esquemas sencillos que son de fácil implementación con BIV. Entrega datos con alta precisión para alcanzar y mantener concentraciones deseadas en sitio efecto, proporcionando una ventaja con respecto a la administración farmacológica de acuerdo con dosis estándar. Dichas concentraciones (según objetivos terapéuticos) han sido reportadas por diversos autores ${ }^{26-31}$.

Hay que resaltar que la mayoría de los procesos de simulación farmacológica están limitados por la variación interindividual y el gran número de variables que alteran la farmacocinética y la farmacodinamia de los medicamentos. Este hecho reduce la precisión de los modelos farmacocinéticos y el desempeño de este y otros algoritmos. Por lo tanto, es imprescindible la evaluación de los efectos individuales. Diversos sistemas de medición de la profundidad anestésica están disponibles en la actualidad y su uso contextualizado puede mejorar ciertos desenlaces para el paciente y reducir el riesgo de eventos indeseables como el recordar intraoperatorio ${ }^{32}$.

Esta investigación no presenta una validación de una predicción farmacológica versus una concentración real (medida en sangre). Recurrimos a la estrategia usada por Lerou et al. ${ }^{23}$, ya utilizada por otros autores, que permitió de forma sencilla 

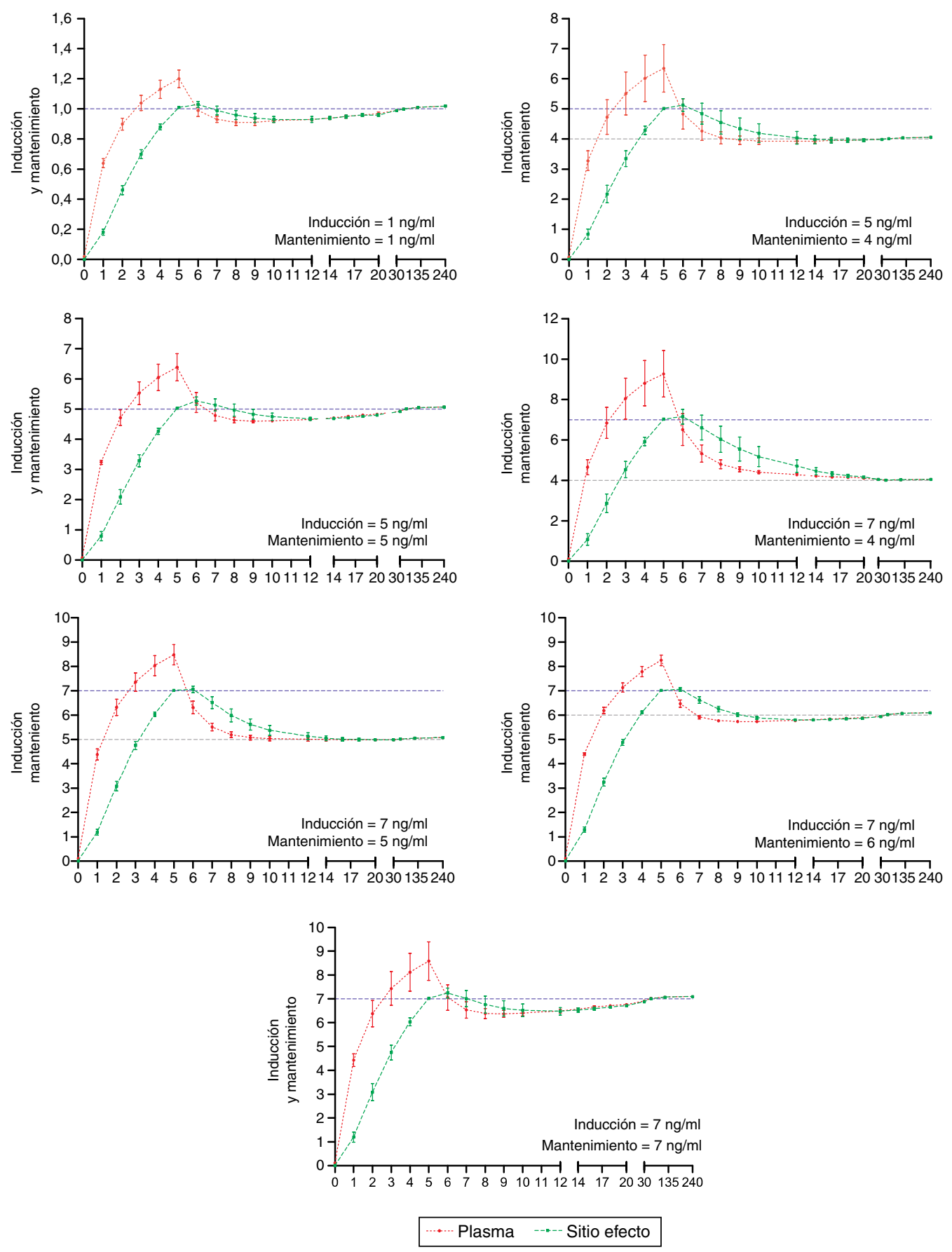

Figura 3 - Concentraciones deseadas y predichas para plasma y sitio efecto organizadas por objetivos deseados para remifentanil.

Fuente: autores.

comparar nuestra predicción matemática (dada por el algoritmo) versus una deseada por el operador. Es de gran interés realizar en el futuro una medición directa de las concentraciones en sangre y compararlas con lo predicho por el modelo propuesto.
Simulación de concentraciones: iTIVA versus TivaTrainer ${ }^{\circledR}$

El algoritmo iTIVA permite obtener en tiempo real las concentraciones en plasma y sitio efecto de los esquemas calculados (de acuerdo con las ecuaciones 1 y 2). Las concentraciones 


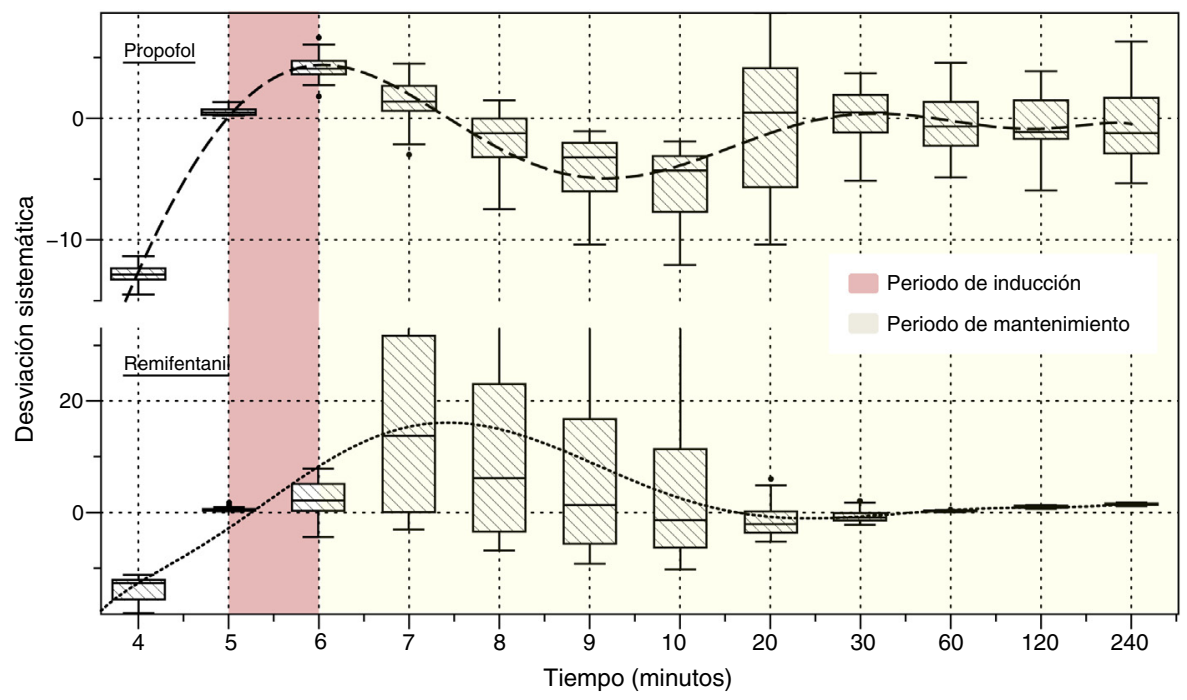

Figura 4 - Desviación sistemática versus tiempo de las concentraciones predichas para propofol y remifentanil por el algoritmo en comparación con las deseadas por el operador.

\section{Fuente: autores.}

obtenidas con iTIVA son iguales a las de TivaTrainer ${ }^{\circledR}$; este último software es utilizado ampliamente en investigación y simulación farmacológica ${ }^{2}$. Este hallazgo, que era esperado puesto que ambas aplicaciones están basadas en el mismo algoritmo matemático, confirma la adecuada implementación del mismo en iTIVA.

\section{Conclusión}

Mediante el uso de modelos farmacocinéticos, la aplicación iTIVA cuantifica las velocidades de infusión necesarias para alcanzar concentraciones en sitio efecto de manera manual para remifentanil y propofol y esto facilita su uso en BIV. Hasta el momento, esta aplicación solo está diseñada para ser una guía durante la administración de infusiones manuales. Su uso no está aprobado como estrategia única de manejo de pacientes y su utilización debe estar orientada y bajo la responsabilidad del anestesiólogo. Este artículo presenta una aproximación teórica basado en un proceso de simulación y su generalización a la práctica clínica requiere de un proceso de validación y comparación frente a otros esquemas de infusión ${ }^{33}$.

\section{Responsabilidades éticas}

Protección de personas y animales. Los autores declaran que para esta investigación no se han realizado experimentos en seres humanos ni en animales.

Confidencialidad de los datos. Los autores declaran que en este artículo no aparecen datos de pacientes.

Derecho a la privacidad y consentimiento informado. Los autores declaran que en este artículo no aparecen datos de pacientes.

\section{Financiamiento}

Este trabajo contó con el apoyo de la Sociedad Colombiana de Anestesiología y Reanimación SCARE.

\section{Conflicto de intereses}

Ninguno declarado.

REFERENCIAS

1. Durieux ME. Anesthetic neurotoxicity: It's not just for children anymore. Anesth Analg. 2010;110:291-2.

2. Struys MM, de Smet T, Mortier EP. Simulated drug administration: An emerging tool for teaching clinical pharmacology during anesthesiology training. Clin Pharmacol Ther. 2008;84:170-4.

3. Muñoz L, Arévalo JJ, Reyes LE, Balaguera CE. Remifentanilo versus propofol con infusión controlada a objetivo en sitio efecto para la sedación de pacientes durante procedimientos endoscópicos gastrointestinales: ensayo clínico controlado aleatorizado. Rev Colomb Anestesiol. 2013;41:114-9.

4. Schnider TW, Minto CF, Shafer SL, Gambus PL, Andresen C, Goodale DB, et al. The influence of age on propofol pharmacodynamics. Anesthesiology. 1999;90:1502-16.

5. Minto CF, Schnider TW, Egan TD, Youngs E, Lemmens HJ, Gambus PL, et al. Influence of age and gender on the pharmacokinetics and pharmacodynamics of remifentanil. I. Model development. Anesthesiology. 1997;86:10-23.

6. Leslie K, Clavisi O, Hargrove J. Target-controlled infusion versus manually-controlled infusion of propofol for general anaesthesia or sedation in adults. Cochrane Database Syst Rev. 2008:CD006059.

7. Masui K, Upton RN, Doufas AG, Coetzee JF, Kazama T, Mortier EP, et al. The performance of compartmental and physiologically based recirculatory pharmacokinetic models for propofol: A comparison using bolus, continuous, and target-controlled infusion data. Anesth Analg. 2010;111:368-79. 
8. Moerman AT, Herregods LL, de Vos MM, Mortier EP, Struys MM. Manual versus target-controlled infusion remifentanil administration in spontaneously breathing patients. Anesth Analg. 2009;108:828-34.

9. Gómez Oquendo FJ, Casas Arroyave FD, Fernández JM, Guarín Grisales A. Anestesia total intravenosa en un sistema de lazo cerrado: reporte del primer caso en Colombia. Rev Colomb Anestesiol. 2013;41:306-10.

10. De Castro V, Godet G, Mencia G, Raux M, Coriat P. Target-controlled infusion for remifentanil in vascular patients improves hemodynamics and decreases remifentanil requirement. Anesth Analg. 2003;96:33-8.

11. Hill M, Peat W, Courtman S. A national survey of propofol infusion use by paediatric anaesthetists in Great Britain and Ireland. Paediatr Anaesth. 2008;18:488-93.

12. Muller T, Ludwig A, Biro P. Two distinct application habits for propofol: An observational study. Eur J Anaesthesiol. 2010;27:265-9.

13. Simpson RB, Russell D. Anaesthesia for daycase gynaecological laparoscopy: A survey of clinical practice in the United Kingdom. Anaesthesia. 1999;54:72-6.

14. Minai FN, Siddiqui KM, Qureshi R. Sedation-analgesia in non operative locations: Practice trends of anaesthetists. J Pak Med Assoc. 2008;58:84-5.

15. Nora FS, Aguzzoli M, Oliveira Filho GR. Current attitude of anesthesiologists and anesthesiology residents regarding total intravenous anesthesia. Rev Bras Anestesiol. 2006;56:362-9.

16. Drews FA, Syroid N, Agutter J, Strayer DL, Westenskow DR. Drug delivery as control task: Improving performance in a common anesthetic task. Hum Factors. 2006;48:85-94.

17. Syroid ND, Agutter J, Drews FA, Westenskow DR, Albert RW, Bermudez JC, et al. Development and evaluation of a graphical anesthesia drug display. Anesthesiology. 2002;96:565-75.

18. Kennedy RR. Seeing the future of anesthesia drug dosing: Moving the art of anesthesia from impressionism to realism. Anesth Analg. 2010;111:252-5.

19. Gin T. Clinical pharmacology on display. Anesth Analg. 2010;111:256-8.

20. Guarracino F, Lapolla F, Cariello C, Danella A, Doroni L, Baldassarri R, et al. Target controlled infusion: TCI. Minerva Anestesiol. 2005;71:335-7.

21. Shafer SL, Siegel LC, Cooke JE, Scott JC. Testing computer-controlled infusion pumps by simulation. Anesthesiology. 1988;68:261-6.
22. R Development Core Team (2008). R: A language and environment for statistical computing. R Foundation for Statistical Computing, Vienna, Austria [consultado 1 Nov 2015]. Disponible en: http://www.R-project.org

23. Lerou JG, Booij LH. Model-based administration of inhalation anaesthesia. 3. Validating the system model. Brit J Anaesth. 2002;88:24-37.

24. Kern SE, Xie G, White JL, Egan TD. A response surface analysis of propofol-remifentanil pharmacodynamic interaction in volunteers. Anesthesiology. 2004;100:1373-81.

25. Thompson JP, Rowbotham DJ. Remifentanil —an opioid for the 21st century. Brit J Anaesth. 1996;76:341-3.

26. Chang CH, Lee JW, Choi JR, Shim YH. Effect-site concentration of remifentanil to prevent cough after laryngomicrosurgery. Laryngoscope. 2013;123:3105-9.

27. Kim EJ, Shin SW, Kim TK, Yoon JU, Byeon GJ, Kim HJ. The median effective effect-site concentration of remifentanil for minimizing the cardiovascular changes to endotracheal intubation during desflurane anesthesia in pediatric patients. Korean J Anesthesiol. 2012;63:314-20.

28. Lee JY, Yang H, Choi SH, Shin DW, Hong SK, Chun DH. The optimal effect-site concentration of remifentanil to attenuate the pain caused by propofol. Korean J Anesthesiol. 2012;63:108-12.

29. Kwak HJ, Min SK, Kim DH, Kang M, Kim JY. Effect-site concentration of remifentanil for nasotracheal versus orotracheal intubation during target-controlled infusion of propofol. J Int Med Res. 2011;39:1816-23.

30. Lee B, Lee JR, Na S. Targeting smooth emergence: The effect site concentration of remifentanil for preventing cough during emergence during propofol-remifentanil anaesthesia for thyroid surgery. Brit J Anaesth. 2009;102:775-8.

31. Albertin A, Casati A, Federica L, Roberto V, Travaglini V, Bergonzi P, et al. The effect-site concentration of remifentanil blunting cardiovascular responses to tracheal intubation and skin incision during bispectral index-guided propofol anesthesia. Anesth Analg. 2005;101:125-30.

32. Bruhn J, Myles PS, Sneyd R, Struys MM. Depth of anaesthesia monitoring: What's available, what's validated and what's next? Brit J Anaesth. 2006;97:85-94.

33. Roberts FL, Dixon J, Lewis GT, Tackley RM, Prys-Roberts C. Induction and maintenance of propofol anaesthesia. A manual infusion scheme. Anaesthesia. 1988;43 Suppl:14-7. 